\title{
Planimetric Features Generalization for the Production of Small-Scale Map by Using Base Maps and the Existing Algorithms
}

\author{
M. Modiri ${ }^{\text {a }}$, M. Mohebbi ${ }^{\text {b }}$, M. Masoumi ${ }^{\text {b }}$, H. Khanlu ${ }^{\text {c, }}$, A. Eftekhari ${ }^{\text {d }}$ \\ ${ }^{\text {a }}$ Professor at Malek Ashtar University of Technology, Esfahan, Iran- mmodiri@ut.ac.ir \\ ${ }^{\mathrm{b}}$ M.s degree of Information Technology Management,- (mohebbi@ Ngo-iran.ir, mm.slm.masumi@gmail.com) \\ ${ }^{c}$ B.s degree of Surveying Engineering, hmkhanlu@yahoo.com \\ ${ }^{\mathrm{d}}$ M.s of degree of Remote Sensing, Dept. of surveying and Geomatics engineering, University of Tehran, Tehran, Iran - \\ a.eftekhari.edu@gmail.com
}

KEY WORDS: Planimetric Features, Generalization, Small-Scale Maps, Base Map, NGO Data Model

\begin{abstract}
:
Cartographic maps are representations of the Earth upon a flat surface in the smaller scale than it's true. Large scale maps cover relatively small regions in great detail and small scale maps cover large regions such as nations, continents and the whole globe. Logical connection between the features and scale map must be maintained by changing the scale and it is important to recognize that even the most accurate maps sacrifice a certain amount of accuracy in scale to deliver a greater visual usefulness to its user. Cartographic generalization, or map generalization, is the method whereby information is selected and represented on a map in a way that adapts to the scale of the display medium of the map, not necessarily preserving all intricate geographical or other cartographic details. Due to the problems facing small-scale map production process and the need to spend time and money for surveying, today's generalization is used as executive approach. The software is proposed in this paper that converted various data and information to certain Data Model. This software can produce generalization map according to base map using the existing algorithm. Planimetric generalization algorithms and roles are described in this article. Finally small-scale maps with 1:100,000, 1:250,000 and 1:500,000 scale are produced automatically and they are shown at the end.
\end{abstract}

\section{INTRODUCTION}

Updated Land Cover maps at the various small scale are used as regional foundation of planning, natural resource management, and land use planning schemes and defensive project and so on. For years, the absence of these information resources is an appreciable and necessary.

The oldest known maps were from five thousand years ago and mapping methods and scientific applications, increasingly in the 17th, 18th and 19th centuries were developed(Freund and Schapire 1995). Many countries were covered by national and international mapping programs before World War II but very little information was available about the maps of different areas. Later emerging aerial photography systems and photogrammetric mapping techniques after World War II, and the subsequent invention of the computer in the decades after 1980, a lot of changes in the patterns to the traditional mapping arose(Brassel and Weibel 1988).

Cartographic maps are representations of the Earth upon a flat surface in the smaller scale than it's true(Robinson, Morrison et al. 1995). Large scale maps cover relatively small regions in great detail and small scale maps cover large regions such as nations, continents and the whole globe. Logical connection between the features and scale map must be maintained by changing the scale and it is important to recognize that even the most accurate maps sacrifice a certain amount of accuracy in scale to deliver a greater visual usefulness to its user(Slocum 1999). Map generalization is the method whereby information is selected and represented on a map in a way that adapts to the scale of the display medium of the map, not necessarily preserving all intricate geographical or other cartographic details. Due to the problems facing small-scale map production process and the need to spend time and money for surveying, today's generalization is used as executive approach(Freund and Schapire 1995).

Cartographic generalization is an overarching process that includes polylines, points and polygons features. In recent years the issue of having an automated method to produce generated maps has attracted the attention of Cartographers(majid hamrah 2012). Based on this, several conceptual, theoretical and operations models have been obtained. One of the biggest obstacles to the lack of standards on spatial data and the lack of a comprehensive approach to automate the process. Also there wasn't full understanding of the generalized maps and cartographic rules which traditionally have used them for years and they never find a good solution for computer automation. So far, there is no comprehensive answer to this problem and different organizations continue their activities in this field according to their requirements and abilities. Generalized method of mapping used by various organizations in different countries are numerous and very diverse in recent years and has a direct correlation with intelligence map information.

With regard to National Geographical Organization (NGO) need for the production of small scale maps, in this article a software is designed and implemented to produce generalized maps of basic maps with the scale 1:25,000 and 1: 50,000.

Also, the conceptual software design stages, methods and outputs have been shown in this paper. 


\section{SOFWARE DESIGN}

Now, despite the efforts by NGO and NCC organizations in Iran, 1:25,000 maps cover the country with an independent data model has been produced that can be used as the basis data on production of small scale maps with the generalization method. In the meantime the infrastructure software design should be like Figure 1.

On the other hands, during the past few years, NGO has been trying to digitize 1:50,000 old and paper maps and update them by IRS satellite images that is very effective in order to supply basic maps in production small scale maps by generalization method. Therefore, the following process should be took place in software design, so design of data model consists of the following steps:

- Data model design and layered features at different scales

- Integration and coordination of layers and map feature classes in a common data model

- The definition of maintenance and display any of the map features at different scales

- Define the display symbols and cartography maps at a common method

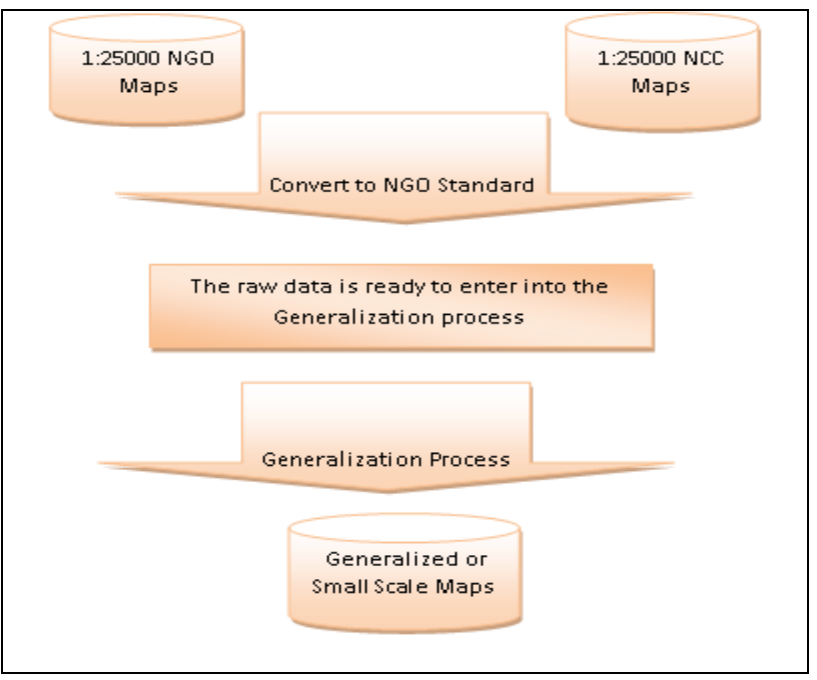

Figure 1. The Stages of base map standardization

\section{METHODOLOGY}

Planimetric generalization include polylines, polygons and points features. Linear features including roads, transmission lines, etc. that removing and simplification algorithms are used to generalization process in the polygons by removing small surface, merging the same level, converting small surface to linear and points features(Liu, Liu et al. 2010). Also in the points features by removing, prioritize, converting points to surface and etc., generalization is completed.

\subsection{Points Features Generalization}

If point's features is too close together and displaying them in a single map is not legible, the points are removed in the proximate locations according to their descriptive property in different forms and a new point is preserved by averaging. Also in some points by considering the significance of their location which cannot be averaged, the points on the real location is chosen and the rest are deleted.

In this method, at the first, all points are selected within circle by a radius proportional to any point, then the selected points are analysis. Finally a one point is preserved as representative of others and the rest of the list are deleted.

An example of spot point's generalization stages is shown in Figure 2.

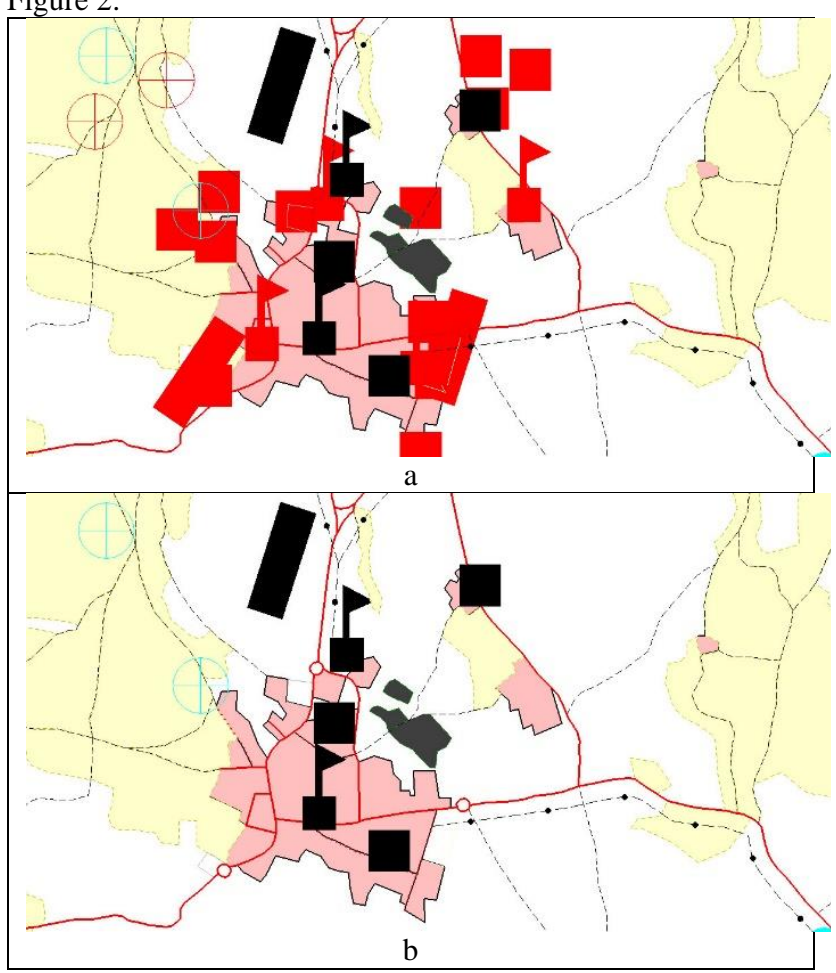

Figure 2. a: selected points are shown, b: deleted selected points and reserved one point.

\subsection{Polygons Generalization}

For generalization polygons, a features with smaller area than threshold are selected and then they are merged together or removed according to cartographer knowledge. Converting small surface to integrated area and smoothing lines are the most important action in the generalization process that removing duplicate lines is used for merging polygons(Bjørke 2003). In the Figure 3, the polygons generalization of garden level is shown that generalized by merging method.

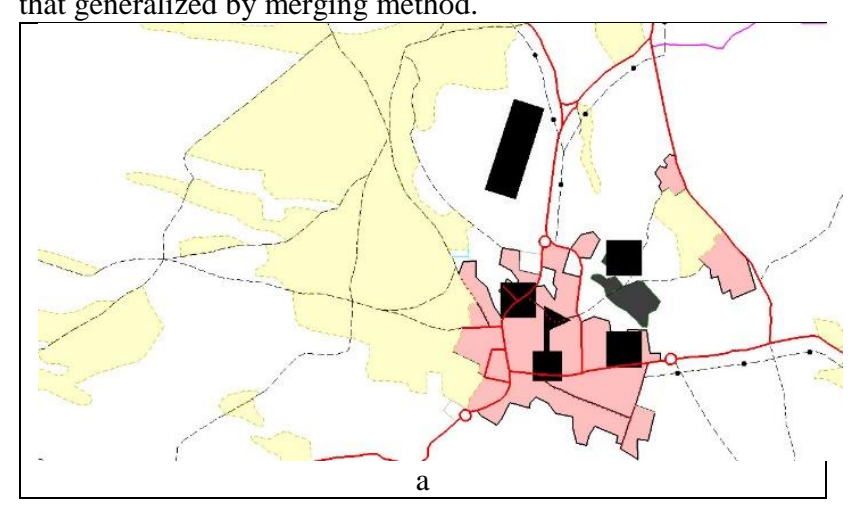




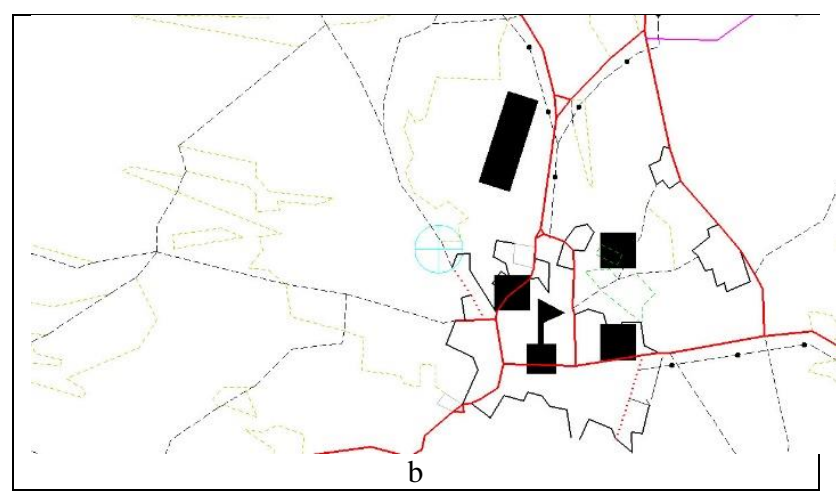

Figure 3. a: polygons before generalization, b: merged polygons after generalization

Some of the surface features, such as residential areas after the collection not available to display in generalized scale map, so they convert to point symbol. For example, residential level with area smaller than 500,000 square meters are converted to points in 1: 250,000 maps. That is shown in Figure 4.
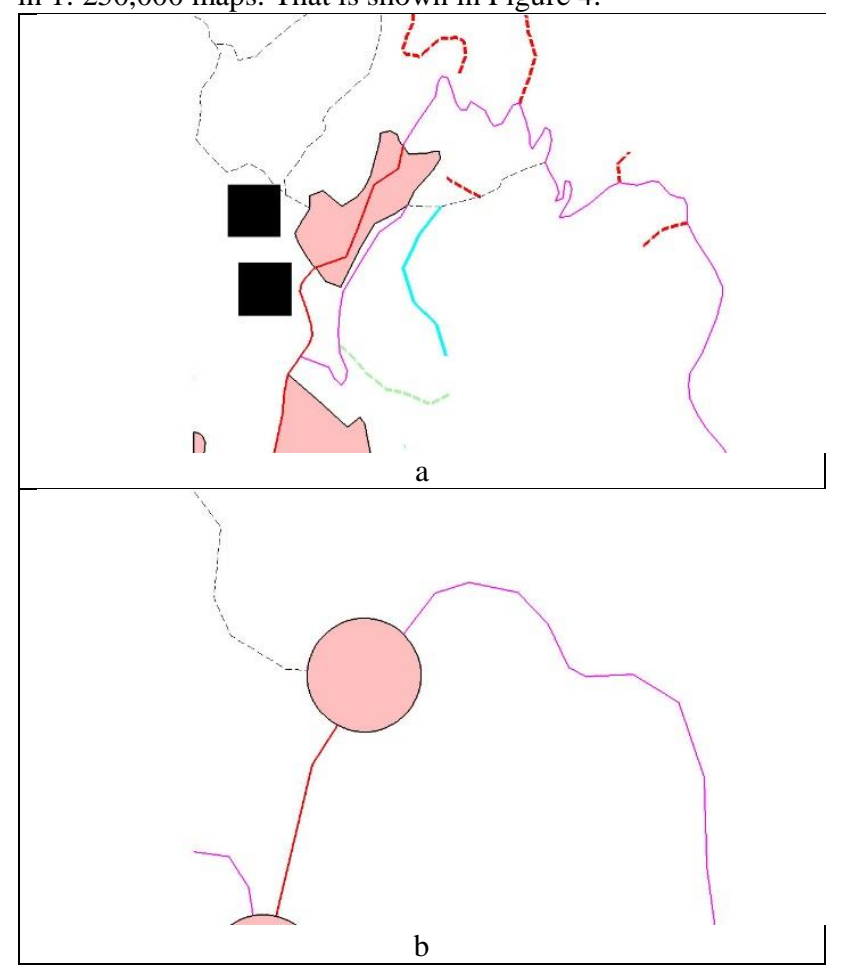

Figure 4. a: small polygons that shown before generalization. $b$ : polygons covert to one point after generalization.

As well as, if the hydrological features as rivers and lakes are not available to display in generalized map, they convert to linear or points features. In this way, the model by maximum removal coefficients are used for deleting. For example, the river by width smaller than 300 meters in 1:250,000 generalized maps are found as duplicate features and merge together. An example of this operation has been shown in Figure 5.
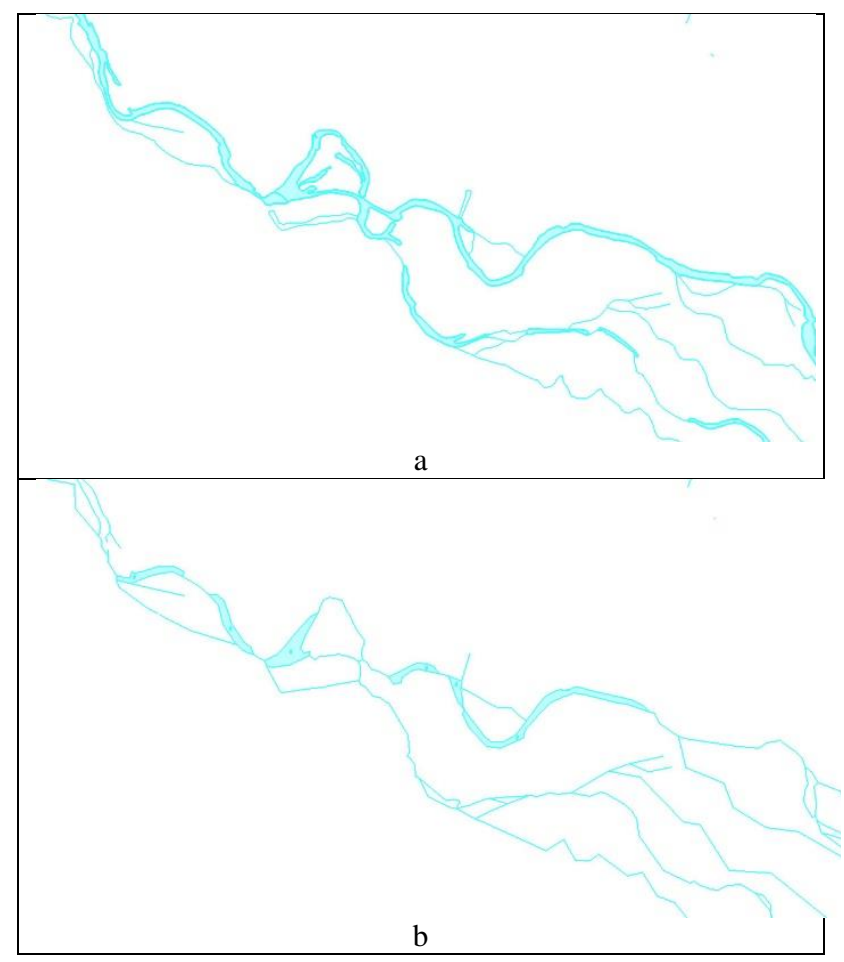

Figure 5. a: the river in base map is shown by polygon, b: the river convert to polyline in generalized map

\subsection{Linear Features Generalization}

The roads and rivers are the most linear features removed in generalization process. Maintaining logical connection between roads, considering the responsible organization to build and maintain roads, classification road parts as highway, expressway and asphalted, selecting the shortest path and having best of view in roads, are the notification cases should be considerate by cartographer when they removed the roads in generalization process(Bjørke 2004). Also after any changing in the road networks, their topology is updated automatically. In the generalization software design lines program is run in different parameters depending on the linear features density of the map. For example, in the rivers generalization, smaller lines by 250 meters length in the first parameter, 500 and 1000 meters in the next parameter to 1: 50,000 maps are selected to delete.

An important part of linear generalization is deleting extra points in the line and arc breaks according to map scale. In which case the volume is low but that is caused irrational fractures in outlines and the beauty of the map doesn't keep. To fix this problem in the design of the software, softening rules has been used in the outlines. These changes are applied in three groups: the road, land cover levels and the contours with hydrological features.

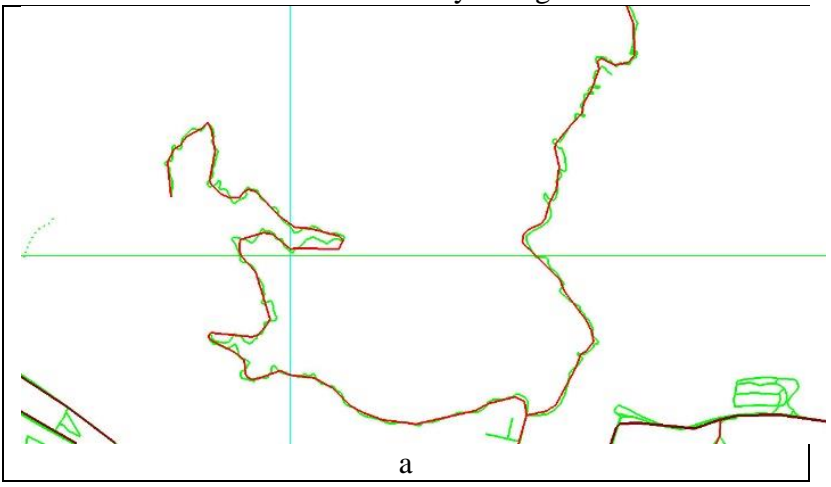




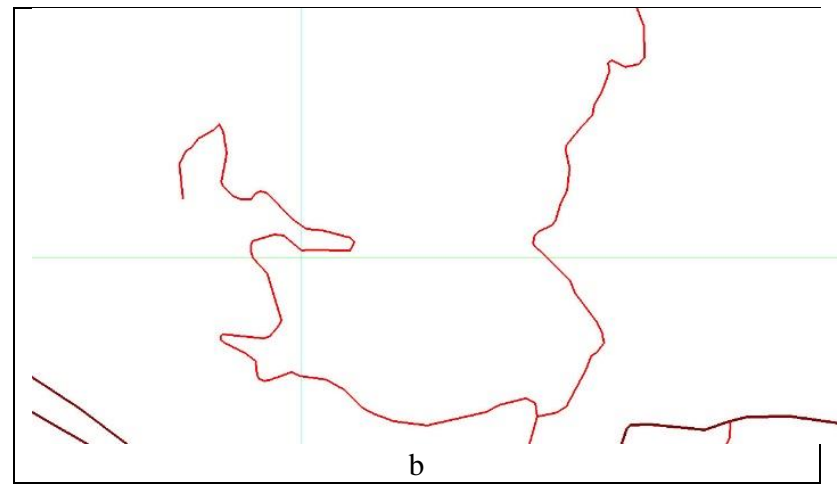

Figure 6. a: linear feature before smoothing. b: linear feature after smoothing

\section{IMPLEMENTATION AND RESULTS}

By applying the algorithm explained in this article, a new module are developed and added to Microstation software that this module has ability to produce 1:50,000, 1:100,000, 1:250,000 and 1:500,000 maps by generalization method. The module is shown at Figure 8.

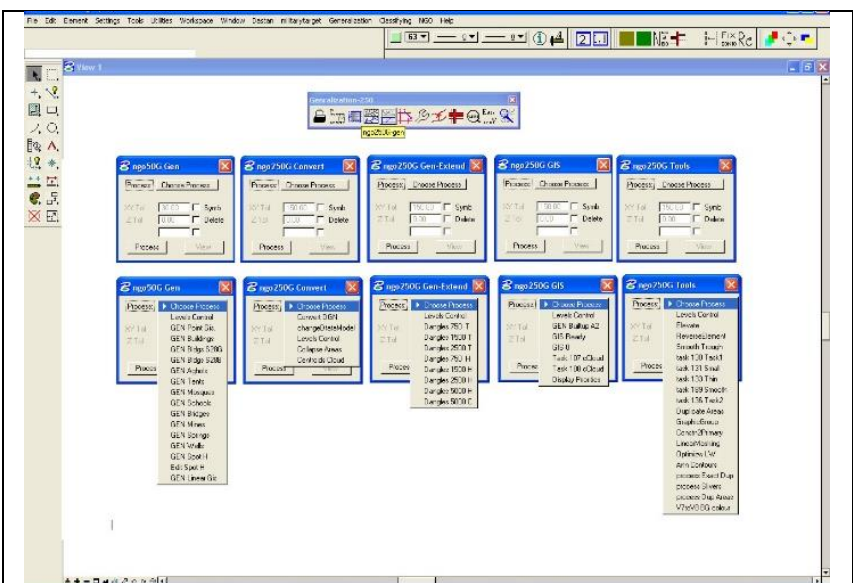

Figure 7. A new Generalization module developed by NGO in Microstation Software

In this software, the various stages of the generalization such as Conversion between any files, eliminating spot points, linear features generalization as roads and rivers, conversion or modulation of polygons, smoothing linear and surface features and etc. are done automatically and by supervision of a cartographer.

In the Figure 9, a sample of generalized maps from 1:25,000 scale in $1: 50,000,1: 100,000,1: 250,000$ and 1:500,000 scales are shown.

As in Figure 9 is also marked, there is a very good coordination between the generalized generated maps, also all features have been shown in the different scale maps according to the existing standards in NOG.

It should be noted that the production of small scale maps to the generalization method presented in this article, for a total of 1 : 50,000 maps for IRAN has been done.

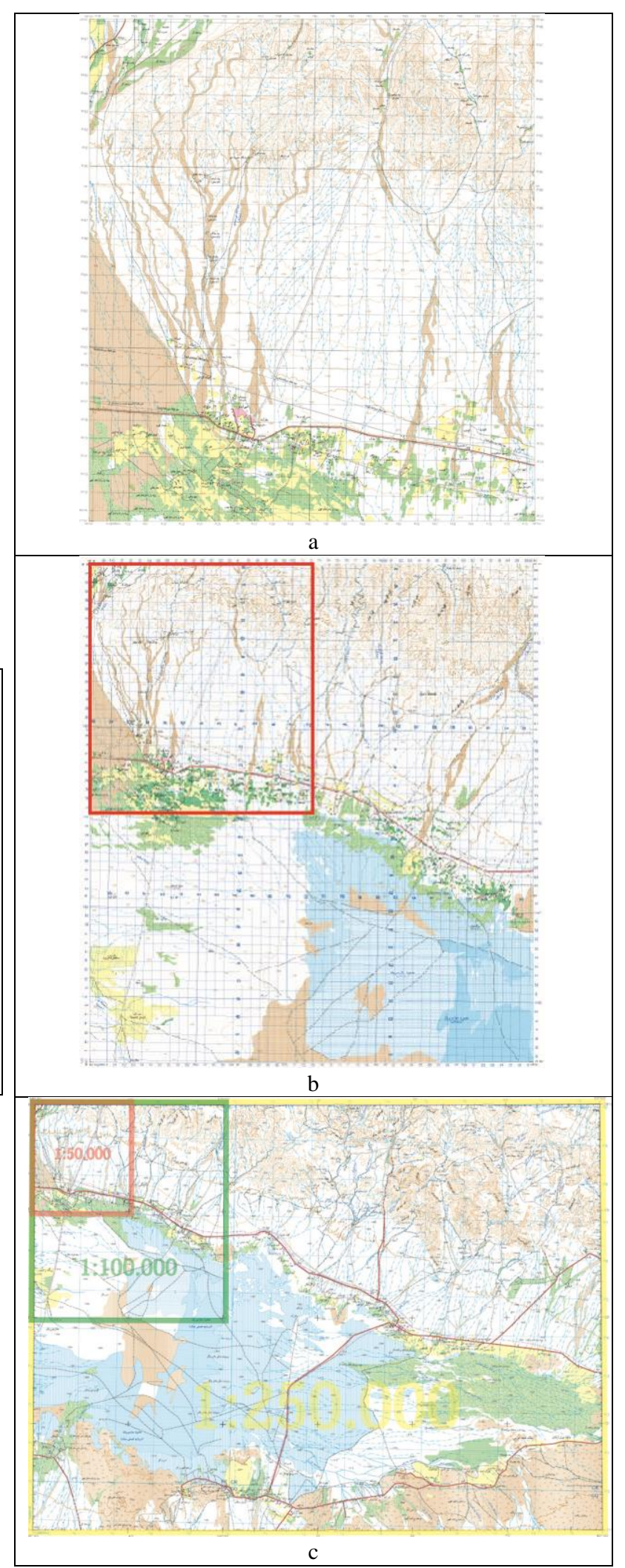




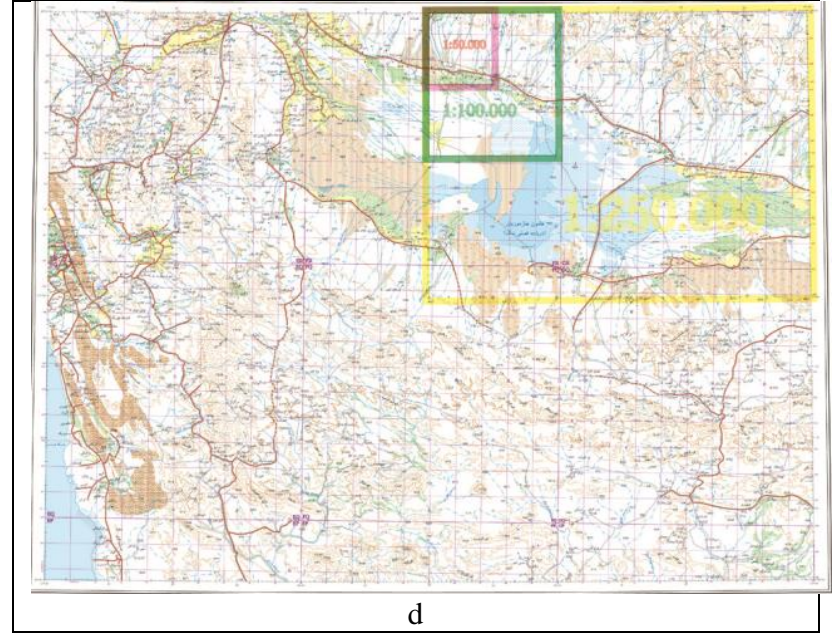

Figure 9. a: 1:50,000 base map. b: 1:100,000 generalized map, c: 1:250,000 generalized map. d: 1:500,000 generalized map.

\section{CONCLUSIONS}

Updated small scale maps are the base maps in regional planning, natural resources management, land and defense plans. Also for years the lack of this information resources for the country has been sensible and needed. Generalization is one of the best and proper methods that used for small scale maps generation from the large scale maps. Planimetric features generalization has been reviewed in this paper. In this regard, the software is designed in accordance with the NGO data model and linear, points and polygons features generalization are done by this software menus. With a small scale map production by automatic generalization method, the maps are produced with considerable speed, the ability to review and update as easily as possible. Of the achievements of generalization project can be pointed to the following:

Achievement geometric and graphical communicating between maps, create a new product (1:100,00 maps), improve the quality of the product, save time and money, keep accuracy and the production of inland and overseas updated maps in various scales.

\section{REFERENCES}

Bjørke, J. (2003). Generalization of road networks for mobile map services: an information theoretic approach. Proceedings of the 21 st International Cartographic Conference (ICC).

Bjørke, J. T. (2004). "Map Generalization of Road Networks." Proceedings of IST-043/RWS-006, Visualisation and the Common Operating Picture: 1-8.

Brassel, K. E. and R. Weibel (1988). "A review and conceptual framework of automated map generalization." International Journal of Geographical Information System 2(3): 229-244.

Freund, Y. and R. E. Schapire (1995). A desicion-theoretic generalization of on-line learning and an application to boosting. Computational learning theory, Springer.

Liu, R., et al. (2010). "A vector generalization of costa's entropypower inequality with applications." Information Theory, IEEE Transactions on 56(4): 1865-1879. majid hamrah, s. j. m. (2012). cartography. Tehran, The research and Development civil House Institute.

Robinson, A. H., et al. (1995). Elements of cartography, Wiley New York.

Slocum, T. A. (1999). Thematic cartography and visualization, Prentice hall Upper Saddle River, NJ. 